\title{
A Two-Stage Procedure for Microwave Imaging of a Buried Dielectric along with the Randomly Rough Surface above It
}

\author{
Tolga Ulaş Gürbüz ${ }^{1}$ and Birol Aslanyürek ${ }^{2}$ \\ ${ }^{1}$ Electrical and Electronics Engineering Faculty, Istanbul Technical University, 34469 Istanbul, Turkey \\ ${ }^{2}$ Department of Mathematical Engineering, Yildiz Technical University, 34210 Istanbul, Turkey \\ Correspondence should be addressed to Tolga Ulaş Gürbüz; tolgaulas@hotmail.com
}

Received 14 October 2014; Accepted 29 January 2015

Academic Editor: Lorenzo Crocco

Copyright @ 2015 T. U. Gürbüz and B. Aslanyürek. This is an open access article distributed under the Creative Commons Attribution License, which permits unrestricted use, distribution, and reproduction in any medium, provided the original work is properly cited.

\begin{abstract}
A two-stage microwave imaging procedure based on the contrast source inversion (CSI) is proposed for the determination of a buried dielectric along with the rough surface above it. It was previously shown that, the CSI, is very effective for the determination of a dielectric buried under a known rough surface. However, for an unknown surface, the application of the CSI to the entire region containing both the object and the roughness will yield significantly inaccurate dielectric property values and, thus, determination of objects will be almost impossible especially when they are small in size or low in contrast. Thus, we propose to construct a reference model for the background without the object by preimaging the entire region in a frequency-hopping scheme and imposing the a priori known property values to the approximately determined morphology of the background. In the second stage, the CSI is performed at single frequency, assuming the constructed reference model as the background. In this case, by taking the advantage of nonlinear inversion and without a restrictive assumption about the characteristics of the rough surface, the proposed approach yields qualitatively satisfactory results even for multiple objects buried under a surface having a high frequency or large roughness.
\end{abstract}

\section{Introduction}

The use of microwaves for detection or imaging of buried objects in remote sensing applications such as geophysical exploration, mine detection, and medical imaging, has been the subject of various research studies. In practice, in most of these remote sensing applications, the object to be determined is a dielectric and the surface above it is rough. However, despite its practical importance, very few studies deal with the imaging of dielectrics buried under a rough surface and among them [1-4], assuming the surface above the object to be known. Only a couple of studies are intended to determine both the object and the rough surface above it by using the same data [5-8]. On the other hand, these studies are based on some assumptions related to the rough surface and/or the buried object and thus are subject to the limits of these assumptions. More precisely [5] is based on the small perturbations theory which severely limits the size of the rough surface that can be determined. In $[6,7]$ the rough surface is assumed to be represented as a sum of B-splines and a buried object can be imaged together with relatively lowfrequency and small or moderate roughness above it. Despite its extra computational burden, a fully nonlinear inversion algorithm such as the contrast source inversion (CSI) [9] and the distorted born iterative method (DBIM) [10], in general, sufficiently reconstructs inhomogeneous dielectric properties of scatterers which are relatively large in size and high in contrast by preserving the nonlinear characteristics of the problem. We previously proposed an approach which enables application of the CSI for the determination of a buried dielectric under a known rough surface [4]. Now in this paper, we suggest the use of the CSI also for the detection of the rough surface above the object. However, in most cases, although it is one of the most successful nonlinear inversion techniques, applying the CSI alone in a single step is not sufficient for accurately reconstructing the property values of the entire region that hosts both the object and the rough background. Thus, we propose a two-stage 
procedure whose first stage is dedicated to the construction of a reference model without the object. For this purpose, the CSI is first applied in a frequency hopping scheme similar to the one given in [11] to the entire region that hosts both the rough surface and the object. The dielectric property values reconstructed in this step may be far from the actual ones, and in this case, determination of objects will be almost impossible especially when they are small in size or low in contrast. On the other hand, in the applications given in the literature, it is consistently observed that the results achieved via the CSI enable discrimination of nonzero contrasts from the zero contrasts, that is, the scatterer from the background in a feasible accuracy, even if the contrast values are not reconstructed accurately [12]. Accordingly, the rough surface between two half-spaces can be approximately distinguished despite the inaccurate values of the reconstructed dielectric parameters. Then a reference model without the object is constructed by assigning the a priori known effective permittivity and conductivity values of the ground to the region below the approximately determined rough surface. In the second stage, the CSI is performed at single frequency by choosing the constructed reference model as the background. In this case, the object and the differences of the assumed rough surface from the actual profile can be determined. As a final step, the region of interest can be narrowed to the union of a region around the approximate location observed in the preceding step and a band in the neighborhood of the assumed rough surface. This improves the quality of the results by both enabling application of any constraints about the contrast of the object and minimization of the reconstruction domain. Here, we should mention that we have not been able to attain quantitatively accurate results through the proposed approach even for a narrower domain. Instead, we have obtained results that give the approximate location and geometry of the buried scatterer.

It should also be noted that the retrieval of the rough surface in the first stage of the above procedure could be achieved through some inversion strategies based on high frequency approaches (e.g., geometrical optics). On the other hand, this would probably entail differences between the data acquisition processes of the two stages such as antennas working in different bands. However, in the proposed approach, the data collected through the same configuration can be used in both stages as shown in the numerical simulations.

The organization of the paper is as follows. The statement of the imaging problem is given in Section 2. Section 3 is devoted to some numerical results while conclusions are presented in Section 4.

Throughout the paper, the $e^{(-\mathbf{i} \omega \mathbf{t})}$ time factor is suppressed and vectors are denoted by bold letters.

\section{Statement of the Imaging Problem}

In this study, assuming that the geometry is uniform along the $\mathrm{O} x_{3}$-direction, the problem of imaging an unknown object along with the rough surface above it will be treated as a $2 \mathrm{D}$ problem on the $O x_{1} x_{2}$ plane as shown in Figure 1. Here, two homogeneous half-spaces with constitutive parameters $\varepsilon_{1}, \sigma_{1}$

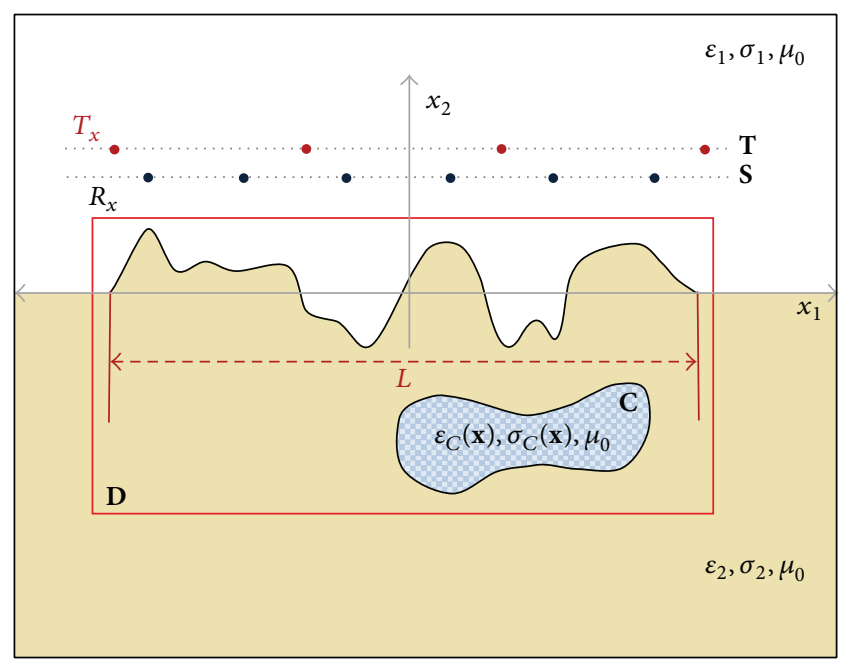

FIgURE 1: Geometry of the problem.

and $\varepsilon_{2}, \sigma_{2}$ are separated by a locally rough interface $\Gamma$ whose coordinates are $\left(x_{1}^{\Gamma}, f\left(x_{1}^{\Gamma}\right)\right)$ with $f$ defined as

$$
f\left(x_{1}^{\Gamma}\right)= \begin{cases}g\left(x_{2}^{\Gamma}\right), & x_{1}^{\Gamma} \in L \\ 0, & x_{1}^{\Gamma} \in \mathbb{R} \backslash L,\end{cases}
$$

where $g$ is a real-valued function and subset $L \subset \mathbb{R}$ is the finite interval over which the interface deviates from a flat surface. Note that $g$ may exhibit jump discontinuities particularly in geological applications where reconstruction of a faulted surface is a common problem. In the lower halfspace, an infinitely long cylindrical object having a crosssection $C$ with the $O x_{1} x_{2}$ plane is located. The dielectric permittivity and the conductivity of the object are $\varepsilon_{C}(\mathbf{x})$ and $\sigma_{C}(\mathbf{x})$ respectively, where $\mathbf{x}=\left(x_{1}, x_{2}\right)$ is the position vector in $\mathbb{R}^{2}$. The magnetic permeabilities of all materials are equal to the vacuum permeability $\mu_{0}$. Region $D$, in which both the object and the rough surface are known to lie, is illuminated individually by a set of time-harmonic line sources located at the points $\mathbf{z}_{j}, j=1,2, \ldots, J$, on line $T$ and, for each illumination, the electromagnetic field that arises from the interaction of the incident field with the object and with the lower half-space is measured on line $S$.

The two-stage imaging procedure given here can be summarized as follows. In the first stage, the rough surface is determined in a frequency-hopping scheme in which the contrast source inversion (CSI) is consecutively performed at $M$ equally spaced frequencies in a frequency band by assuming the results of the previous inversion as the background. Then a reference model without the object is constructed by properly modifying the results achieved at the $M$ th frequency. In the second stage, the buried object is imaged, by using the constructed reference model as the background at an appropriate $(M+1)$ th frequency. The details of the procedure are given below.

In line with the procedure outlined in above, each source illuminates the region at angular frequencies $\omega^{(m)}, m=1$, $2, \ldots, M,(M+1)$. The electric field vector of each incident 
wave is $\mathbf{E}_{i}^{(m)}\left(\mathbf{x} ; \mathbf{z}_{j}\right)=u_{i}^{(m)}\left(\mathbf{x} ; \mathbf{z}_{j}\right) \mathbf{e}_{3}$ where $\mathbf{e}_{3}$ is the unit vector in the $\mathrm{Ox}_{3}$-direction. Then the problem is reduced to a scalar one in terms of the field function $u_{j}^{(m)}(\mathbf{x})$ which represents the total electric field vector $\mathbf{E}^{(\mathbf{m})}\left(\mathbf{x} ; \mathbf{z}_{j}\right)=$ $u^{(\mathbf{m})}\left(\mathbf{x} ; \mathbf{z}_{j}\right) \mathbf{e}_{3}$ in $\mathbb{R}^{2}$ for the illumination of the $j$ th source at the $m$ th frequency. Both the rough surface and the object are attended to be determined by using the same data, namely, the measured field $u_{j}^{(m)}(\mathbf{x})$ for $\mathbf{x} \in S$. This field can be expressed as $u_{j}^{(m)}(\mathbf{x})=u_{b, j}^{(m)}(\mathbf{x})+u_{s, j}^{(m)}(\mathbf{x})$ where $u_{b, j}^{(m)}(\mathbf{x})$ is the background field due to a background model and $u_{s, j}^{(m)}(\mathbf{x})$ is the scattered field due to the differences between the background model and the actual profile. $u_{b, j}^{(m)}(\mathbf{x})$ can be synthetically generated for any assumed background model. Then the imaging problem can be expressed as the extraction of $\chi_{D}^{(m)}(\mathbf{x})$ from the following system of integral equations:

$$
\begin{gathered}
u_{j}^{(m)}(\mathbf{x})=u_{b, j}^{(m)}(\mathbf{x}) \\
+\iint_{D} G_{b}^{(m)}\left(\mathbf{x} ; \mathbf{x}^{\prime}\right) k_{b}^{(m)^{2}}\left(\mathbf{x}^{\prime}\right) \chi_{D}^{(m)} \\
\cdot\left(\mathbf{x}^{\prime}\right) u_{j}^{(m)}\left(\mathbf{x}^{\prime}\right) d \mathbf{x}^{\prime}, \\
\mathbf{x} \in D, \\
u_{s, j}^{(m)}(\mathbf{x})=\iint_{D} G_{b}^{(m)}\left(\mathbf{x} ; \mathbf{x}^{\prime}\right) k_{b}^{(m)^{2}}\left(\mathbf{x}^{\prime}\right) \chi_{D}^{(m)} \\
\cdot\left(\mathbf{x}^{\prime}\right) u_{j}^{(m)}\left(\mathbf{x}^{\prime}\right) d \mathbf{x}^{\prime}, \\
\mathbf{x} \in S,
\end{gathered}
$$

which are the well-known object and data equations, respectively [9]. Here $G_{b}^{(m)}\left(\mathbf{x} ; \mathbf{x}^{\prime}\right)$ is Green's function of the assumed background for the $m$ th frequency whose computation is briefly given in a subsection below. The function $\chi_{D}^{(m)}(\mathbf{x})$ is the contrast function and is defined by

$$
\chi_{D}^{(m)}(\mathbf{x})=\frac{k^{(m)^{2}}(\mathbf{x})}{k_{b}^{(m)^{2}}(\mathbf{x})}-1,
$$

where $k^{(m)}(\mathbf{x})$ and $k_{b}^{(m)}(\mathbf{x})$ are the wave-numbers corresponding to the actual dielectric profile and the assumed background, respectively. The squares of the wave-numbers are given by

$$
\begin{aligned}
& k_{b}^{(m)^{2}(\mathbf{x})} \\
& = \begin{cases}k_{1}^{(m)^{2}}, & \mathbf{x} \notin D, x_{2}>0 \\
k_{2}^{(m)^{2}}, & \mathbf{x} \notin D, x_{2}<0 \\
\omega^{(m)^{2}} \widetilde{\varepsilon}_{D}^{(m-1)}(\mathbf{x}) \mu_{0}+i \omega^{(m)} \widetilde{\sigma}_{D}^{(m-1)}(\mathbf{x}) \mu_{0}, & \mathbf{x} \in D,\end{cases}
\end{aligned}
$$

$$
\begin{aligned}
k^{(m)^{2}}(\mathbf{x}) & \\
\quad & \begin{cases}k_{1}^{(m)^{2}}, & x_{2}>f\left(x_{1}\right) \\
k_{2}^{(m)^{2}}, & x_{2}<f\left(x_{1}\right), \mathbf{x} \notin C \\
\omega^{(m)^{2}} \varepsilon_{c}(\mathbf{x}) \mu_{0}+i \omega^{(m)} \sigma_{c}(\mathbf{x}) \mu_{0}, & \mathbf{x} \in C,\end{cases}
\end{aligned}
$$

with $k_{p}^{(m)^{2}}=\omega^{(m)^{2}} \varepsilon_{p} \mu_{0}+i \omega^{(m)} \sigma_{p} \mu_{0}, p=1$, 2. In (4), $\widetilde{\varepsilon}_{D}^{(m-1)}(\mathbf{x})$ and $\widetilde{\sigma}_{D}^{(m-1)}(\mathbf{x})$ denote the dielectric properties in region $D$ of the assumed background and they are extracted from $\widetilde{\chi}_{D}^{(m-1)}(\mathbf{x})$, namely, the contrast reconstructed in the previous frequency step. The contrast function $\chi_{D}^{(m)}(\mathbf{x})$ cannot be extracted from (2) exactly since it is an ill-posed and nonlinear problem. Instead, an estimated contrast $\widetilde{\chi}_{D}^{(m)}(\mathbf{x})$ can be achieved through an iterative inversion algorithm. We here propose to use the standard CSI method which is an efficient nonlinear inversion algorithm summarized in a subsection below.

The above process is carried out for $m \in[1, M]$ choosing the dielectric properties in region $D$ of the initial background, that is, the background for $m=1$, as $\widetilde{\varepsilon}_{D}^{0}(\mathbf{x})=\varepsilon_{1}$ and $\widetilde{\sigma}_{D}^{0}(\mathbf{x})=$ $\sigma_{1}$.

Although this frequency-hopping scheme significantly improves the performance of the CSI, in most applications, the results achieved at the $M$ th step will not be sufficient to determine the buried object, especially in relatively large domains that host objects small in size or low in contrast. On the other hand, an approximate rough surface $\widetilde{\Gamma}$ between two half-spaces can be determined using $\widetilde{\varepsilon}_{D}^{(M)}(\mathbf{x})$ and $\widetilde{\sigma}_{D}^{(M)}(\mathbf{x})$ profiles despite their incorrect values. This could be achieved even by visual interpretation or by using some edge detection approaches. In the final step of the first stage, a reference model with dielectric properties $\varepsilon_{D, \text { ref }}(\mathbf{x})$ and $\sigma_{D, \text { ref }}(\mathbf{x})$ is constructed by assigning the a priori known effective permittivity and conductivity values of the upper and lower half-spaces, that is $\varepsilon_{1}, \sigma_{1}$ and $\varepsilon_{2}, \sigma_{2}$, to the regions above and below the approximately determined rough surface, respectively.

In the second stage, the buried object is imaged by using the constructed reference model as the background at an appropriate $(M+1)$ th frequency which may but does not have to be in the frequency range of the first stage. More precisely, $\widetilde{\chi}_{D}^{(M+1)}(\mathbf{x})$ is reconstructed via the CSI by using $\varepsilon_{D \text {,ref }}(\mathbf{x})$ and $\sigma_{D \text {,ref }}(\mathbf{x})$ instead of $\widetilde{\varepsilon}_{D}^{(M)}(\mathbf{x})$ and $\widetilde{\sigma}_{D}^{(M)}(\mathbf{x})$. Note that $u_{b, j}^{(M+1)}(\mathbf{x})$ and $G_{b}^{(M+1)}(\mathbf{x} ; \mathbf{y})$ will be numerically obtained for this reference model in this case. Once $\tilde{\chi}_{D}^{(M+1)}(\mathbf{x})$ is achieved, the corresponding $\widetilde{\varepsilon}_{D}^{(M+1)}(\mathbf{x})$ and $\widetilde{\sigma}_{D}^{(M+1)}(\mathbf{x})$ can be calculated considering the reference background model. As an additional step, the reconstruction domain can be narrowed to $\grave{D}=D_{1} \cup D_{2}$ where $D_{1}$ is a region around the approximate location of the object which may be determined through the interpretation of the results of the preceding step and $D_{2}$ is a band in the neighborhood of the approximate rough surface $\widetilde{\Gamma}$. Thiswill improve the quality of the results by both enabling application of some constraints such as 
positivity of the contrast associated with the object separately and minimization of the reconstruction domain.

Note that the change of the dielectric property values of the materials with the frequencies used throughout the given procedure is assumed to be negligible. The flowchart of the overall imaging strategy is given in Figure 2.

2.1. The Estimation of the Contrast via the CSI. In (2), both $\chi_{D}^{(m)}$ and $u_{j}^{(m)}$ are the unknowns. Note that the total field $u_{j}^{(m)}$ itself is a function of $\chi_{D}^{(m)}$, which makes the problem nonlinear in terms of $\chi_{D}^{(m)}$. The CSI method offers defining the induced current $w_{j}^{(m)}=\chi_{D}^{(m)} u_{j}^{(m)}$ as the "contrast source" and rewriting (2) for the contrast source as

$$
\begin{gathered}
u_{j}^{(m)}=u_{b, j}^{(m)}+G_{D}^{(m)} w_{j}^{(m)}, \quad j=1,2, \ldots, J \\
u_{s, j}^{(m)}=G_{S}^{(m)} w_{j}^{(m)}, \quad j=1,2, \ldots, J .
\end{gathered}
$$

The integral operators $G_{D}^{(m)}$ and $G_{S}^{(m)}$ in symbolically given (6) and (7) are

$$
\left(G_{D, S}^{(m)} \psi\right)(\mathbf{x})=\iint_{D} G_{b}^{(m)}\left(\mathbf{x} ; \mathbf{x}^{\prime}\right) k_{b}^{(m)^{2}}\left(\mathbf{x}^{\prime}\right) \psi\left(\mathbf{x}^{\prime}\right) d \mathbf{x}^{\prime},
$$

$$
\mathbf{x} \in D, S \text {. }
$$

In the standard CSI, the contrast sources and the contrast function that minimize a cost functional are sought iteratively in an alternating manner without applying an extra regularization. This cost functional consists of two terms: sums of the residual norms in both object equation (6) and data equation (7) for each illumination. The explicit expression of the cost functional is

$$
\begin{aligned}
F^{(m)}= & \frac{\sum_{j}\left\|u_{s, j}^{(m)}-G_{S}^{(m)} w_{j}^{(m)}\right\|_{S}^{2}}{\sum_{j}\left\|u_{s, j}^{(m)}\right\|_{S}^{2}} \\
& +\frac{\sum_{j}\left\|\chi_{D}^{(m)} u_{b, j}^{(m)}-w_{j}^{(m)}+\chi_{D}^{(m)} G_{D}^{(m)} w_{j}^{(m)}\right\|_{D}^{2}}{\sum_{j}\left\|\chi_{D}^{(m)} u_{b, j}^{(m)}\right\|_{D}^{2}},
\end{aligned}
$$

where $\|\cdot\|_{S}^{2}$ and $\|\cdot\|_{D}^{2}$ denote the norms on $L_{2}(S)$ and $L_{2}(D)$, respectively. We refer the reader to [9] for the details of the iterative process.

\subsection{Green's Function of the Assumed Background. Green's} function of the assumed background, $G_{b}^{(m)}\left(\mathbf{x} ; \mathbf{x}^{\prime}\right)$, can be obtained by separating it into two components as

$$
G_{b}^{(m)}\left(\mathbf{x} ; \mathbf{x}^{\prime}\right)=G_{0}^{(m)}\left(\mathbf{x} ; \mathbf{x}^{\prime}\right)+G_{12}^{(m)}\left(\mathbf{x} ; \mathbf{x}^{\prime}\right) .
$$

Here, $G_{12}^{(m)}\left(\mathbf{x} ; \mathbf{x}^{\prime}\right)$ is Green's function of the two-layered media with a planar interface. This function, in general, is expressed in terms of infinite integrals and a method based on the two-level Discrete Complex Images Method [13] is given in [4] for the efficient calculation of it. Then $G_{0}^{(m)}\left(\mathbf{x} ; \mathbf{x}^{\prime}\right)$ can

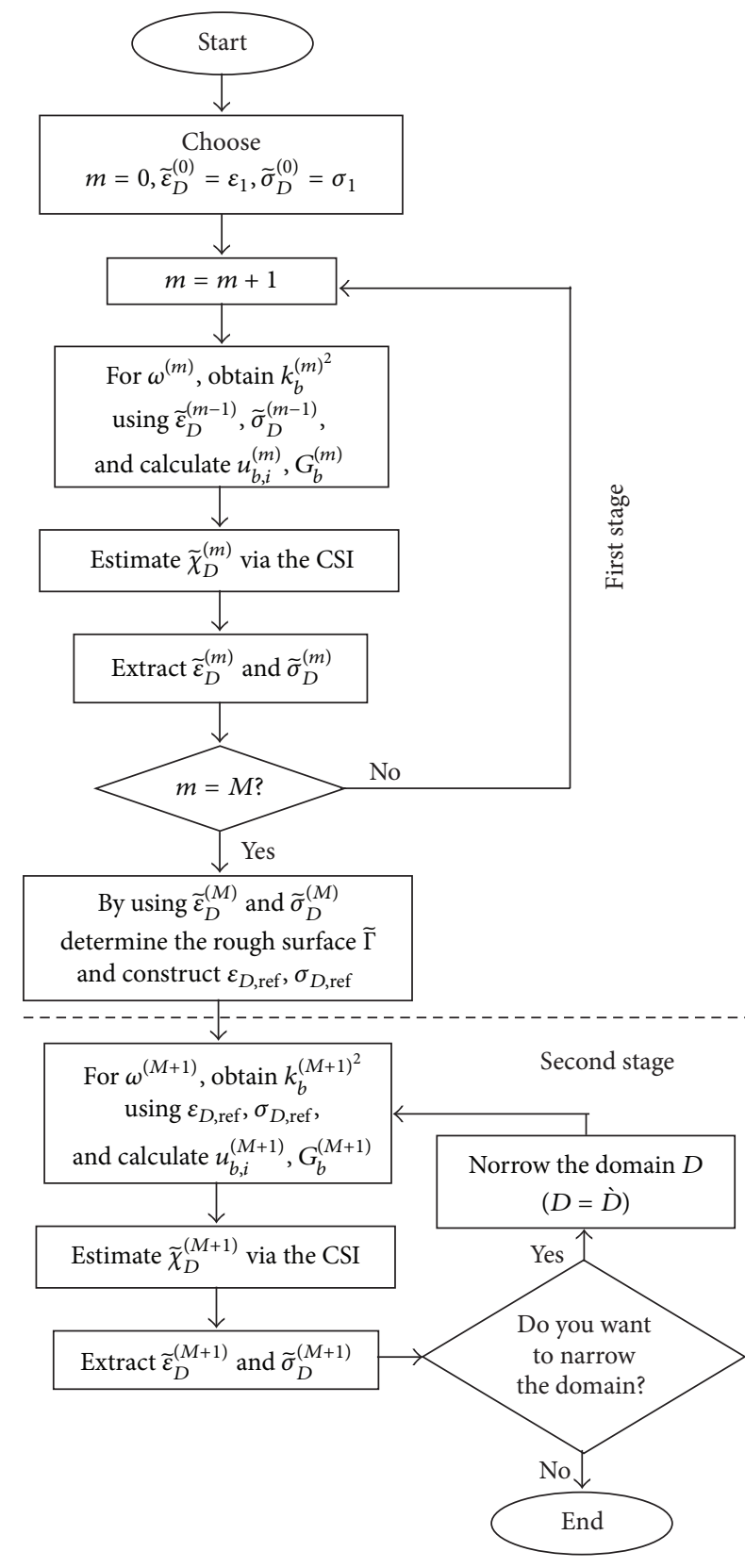

FIGURE 2: The flowchart of the two-stage imaging procedure.

be considered as the scattered field due to the differences between the assumed background and the planarly layered media for a line source of unit strength located at point $\mathbf{x}^{\prime}$ and satisfies the following integral equation:

$$
\left(I-Q^{(m)}\right) G_{0}^{(m)}\left(\mathbf{x} ; \mathbf{x}^{\prime}\right)=Q^{(m)} G_{12}^{(m)}\left(\mathbf{x} ; \mathbf{x}^{\prime}\right),
$$

where the integral operator $Q^{(m)}$ is defined by

$$
\begin{aligned}
& \left(Q^{(m)} G\right)\left(\mathbf{x} ; \mathbf{x}^{\prime}\right) \\
& \quad=\iint_{D} G_{12}^{(m)}(\mathbf{x} ; \mathbf{z})\left(k_{b}^{(m)^{2}}(\mathbf{z})-k_{12}^{(m)^{2}}(\mathbf{z})\right) G\left(\mathbf{z} ; \mathbf{x}^{\prime}\right) d \mathbf{z} .
\end{aligned}
$$




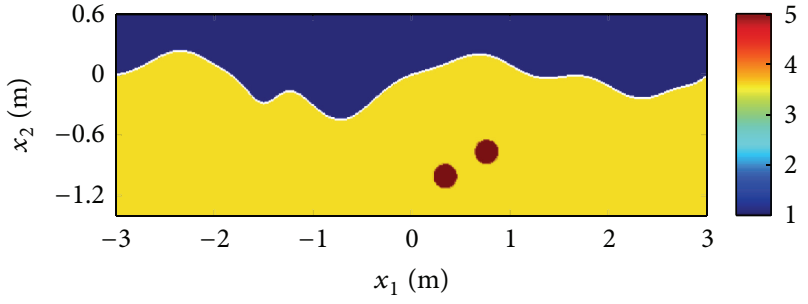

(a)

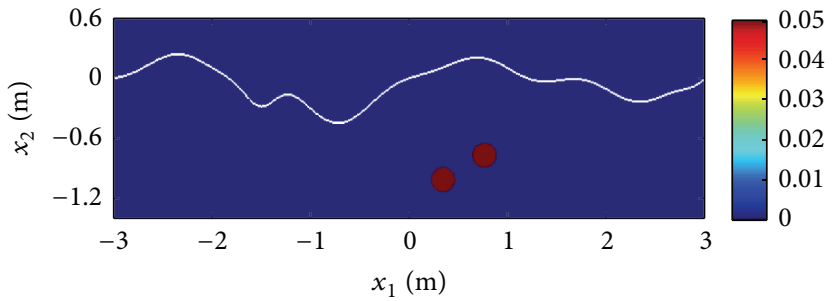

(b)

FIGURE 3: (a) The exact relative permittivity and (b) conductivity profiles of two circular objects and the background.

In (12), $k_{12}^{(m)}(\mathbf{x})$ is the wave-number of the two-layered media with a planar interface and its square is given by

$$
k_{12}^{(m)^{2}}(\mathbf{x})= \begin{cases}k_{1}^{(m)^{2}}, & x_{2}>0 \\ k_{2}^{(m)^{2}}, & x_{2}<0 .\end{cases}
$$

Equation (11) can be solved for $G_{0}^{(m)}\left(\mathbf{x} ; \mathbf{x}^{\prime}\right)$ by applying the forward solution procedure given in [14].

\section{Numerical Simulations}

The proposed approach has been tested through several numerical simulations by using a regular PC with a CPU at 2.00 GHz and $4 \mathrm{~GB}$ RAM. In each simulation, considering the size of the roughness and the object, the penetration depth, and the computational size of the problem, the operating frequencies are chosen as $f^{(m)}=50+(m-1) \times 25, m=$ $1,2, \ldots, 9 \mathrm{MHz}$, in the first stage and, unless otherwise stated, the second stage is performed at $f=150 \mathrm{MHz}$. Note that the number of frequencies in the first stage has been empirically determined as 9 , since it has been observed that increasing the number of frequencies does not significantly improve the results for the given examples. The sizes in the simulations are also given in terms of free space wavelength $\lambda$ at $150 \mathrm{MHz}$. Ten equally spaced line sources located on the line $x_{2}=$ $1 \mathrm{~m}$ are used to illuminate the entire region and, for each illumination, the total field data are synthetically generated on the line $x_{2}=0.9 \mathrm{~m}$ at 40 equidistant points by solving the associated direct scattering problem via the finite element method (FEM). The total field is corrupted with $1 \%$ random noise by adding a random term $\eta\left|u_{j}\right| e^{i 2 \pi r_{d}}$, where $\eta$ is the noise level and $r_{d}$ is a uniformly distributed random variable between 0 and 1 . The corresponding SNR is $-20 \log _{10} \eta=$ $40 \mathrm{~dB}$.

In all simulations, the same reconstruction domain $D$, whose dimensions are $6 \mathrm{~m} \times 2 \mathrm{~m},(3 \lambda \times \lambda)$, is discretized into 4800 cells of size $5 \mathrm{~cm} \times 5 \mathrm{~cm}(0.005 \lambda \times 0.005 \lambda)$. At each frequency, the CSI, which is an iterative process with an error reducing nature, is stopped when the convergence criterion is satisfied; namely, the difference between the cost functionals of two consecutive iterations is smaller than $10^{-7}$. The maximum number of iterations is chosen as 1000 and the process is terminated when it is reached without satisfying the convergence criterion. The corresponding computational time for the entire procedure including the calculation of
Green's functions and the iterative process is about $9 \mathrm{~min}$ at each frequency.

In the first simulation, as seen in Figure 3, two identical circular objects with radius $0.12 \mathrm{~m}(0.06 \lambda)$ are buried under a rough surface, which is plotted in white. Here, the lower halfspace is composed of a low-loss material that may represent dry soil with relative permittivity $\varepsilon_{r, 2}=3.6$ and conductivity $\sigma_{2}=10^{-5} \mathrm{~S} / \mathrm{m}$. Both objects, with depths of $1 \mathrm{~m}(0.5 \lambda)$ and $0.76 \mathrm{~m}(0.38 \lambda)$ below the surface $x_{2}=0$, have relative permittivity of $\varepsilon_{r, C}=5$ and conductivity of $\sigma_{C}=0.05$ which constitute a low contrast between the objects and the background. First of all, it is worth showing that applying the CSI alone is not sufficient for accurately reconstructing the property values of the entire region including both the objects and the rough background in a single step. As seen in Figure 1, the region outside domain $D$ consists of two halfspaces with a planar interface. Thus, the natural choice of the background for the inversion procedure is the two-layered media with a planar interface. We have performed the CSI in different frequencies from $50 \mathrm{MHz}$ to $200 \mathrm{MHz}$ and could not be able to obtain any satisfactory results even for the rough surface. The reconstructed relative permittivity and conductivity distributions in the reconstruction domain $D$ at $150 \mathrm{MHz}$ are given in Figures 4(a) and 4(b), respectively.

When we apply the proposed procedure to the same configuration, the unmodified results given in Figures 5(a) and 5(b) are achieved at the end of the frequency-hopping scheme. Note that the boundary of the lower half-space, that is, the unknown rough surface, can be approximately determined while the objects are entirely undetectable. Although visually apparent boundary between two half-spaces can be properly distinguished by some edge detection algorithms, in the examples given here we empirically determined the approximate rough surface $\widetilde{\Gamma}$ by considering the regions whose relative permittivity values are lower than 1.5 to be free space and the others to be the ground. Then the piecewise-homogeneous reference model constructed using $\widetilde{\Gamma}$, which is plotted in red in the figures, is taken as the background for the second stage. The results obtained at $150 \mathrm{MHz}$ are given in Figures 5(c) and 5(d). Here the objects are barely detectable in the permittivity profile while they can be successfully distinguished in the conductivity profile although the values are underestimated. According to these results, the reconstruction domain has been narrowed to the union of two subregions whose boundaries are shown in 


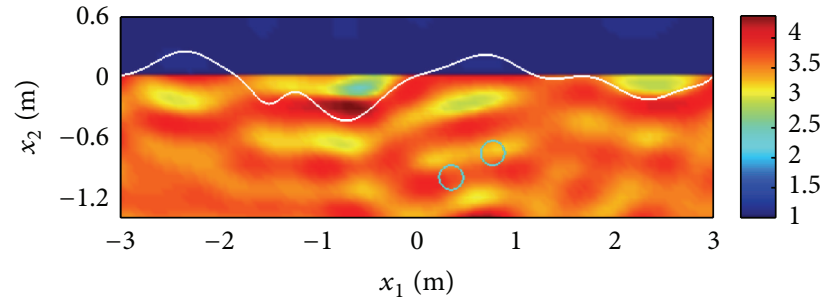

(a)

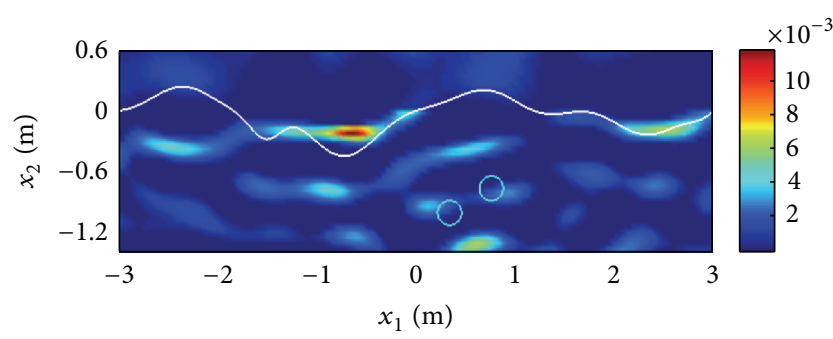

(b)

FIGURE 4: (a) Reconstructed permittivity and (b) conductivity profiles for the direct application of the CSI on a planarly layered background at $150 \mathrm{MHz}$.

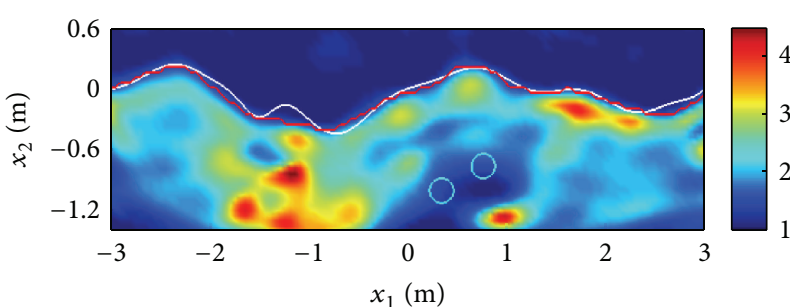

(a)

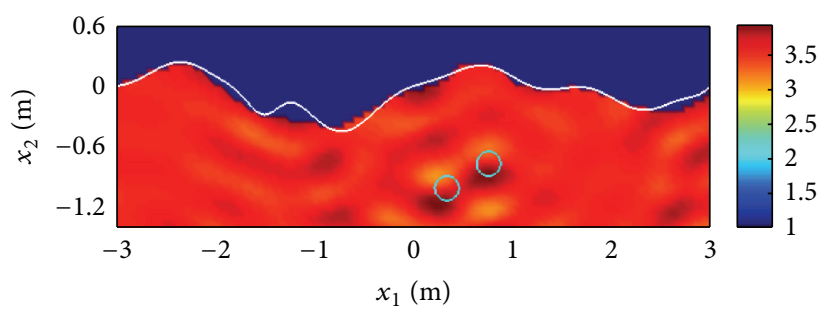

(c)

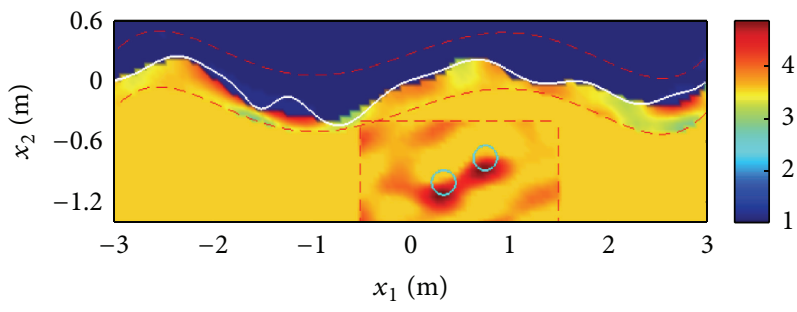

(e)

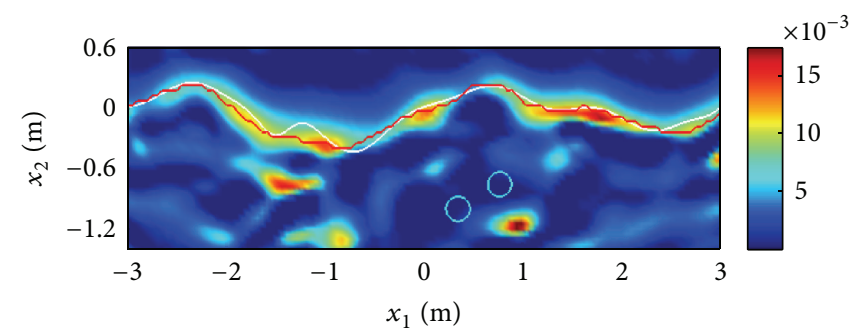

(b)

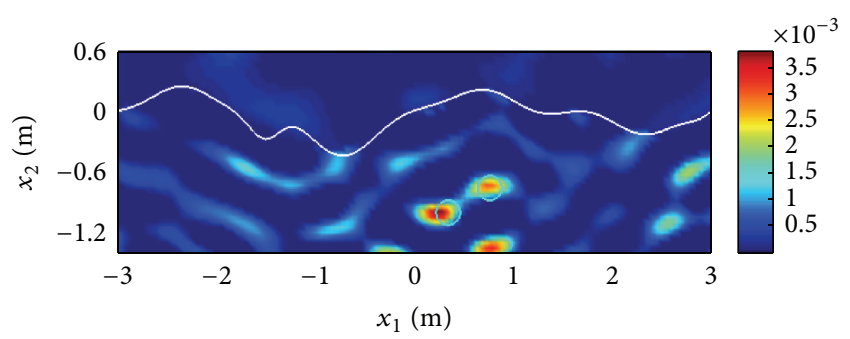

(d)

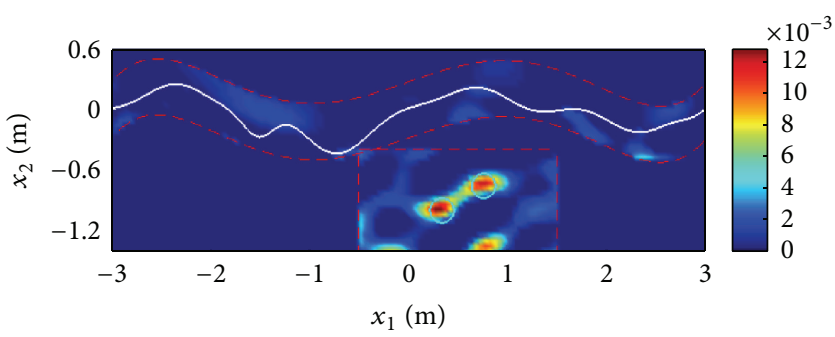

(f)

FiguRE 5: (a) Reconstructed permittivity and (b) conductivity profiles and the determination of the rough surface after the first stage. Final results of permittivity and conductivity profiles ((c)-(d)) for the entire domain and ((e)-(f)) for the narrowed domain.

the dashed lines in Figures 5(e) and 5(f). These subregions consist of a rectangle with dimension of $1 \times 2 \mathrm{~m}(0.5 \lambda \times \lambda)$ and a band whose width is about $0.55 \mathrm{~m}(0.28 \lambda)$. Then the CSI has been performed by applying the positivity constraint for the part around the object. In this case, two close objects can be distinguished with higher property values in both permittivity and conductivity profiles. This improvement is considered to be due to the reduction of the size of the domain and the application of the positivity constraint for the object.
In order to test the effect of the size of the roughness, a larger rough surface is considered, and three circular objects with radius $0.12 \mathrm{~m}(0.06 \lambda)$ are buried under it as shown in Figures 6(a) and 6(b). The dielectric property values of the objects are chosen as $\varepsilon_{r, C}=5$ and $\sigma_{C}=0.05 \mathrm{~S} / \mathrm{m}$ for the one with depth of $1 \mathrm{~m}(0.5 \lambda), \varepsilon_{r, C}=6$ and $\sigma_{C}=0.06 \mathrm{~S} / \mathrm{m}$ for the one with depth of $0.8 \mathrm{~m}(0.4 \lambda)$, and $\varepsilon_{r, C}=7$ and $\sigma_{C}=0.07 \mathrm{~S} / \mathrm{m}$ for the one with depth of $0.6 \mathrm{~m}(0.3 \lambda)$. Similar to the previous example, the objects are undetectable while 


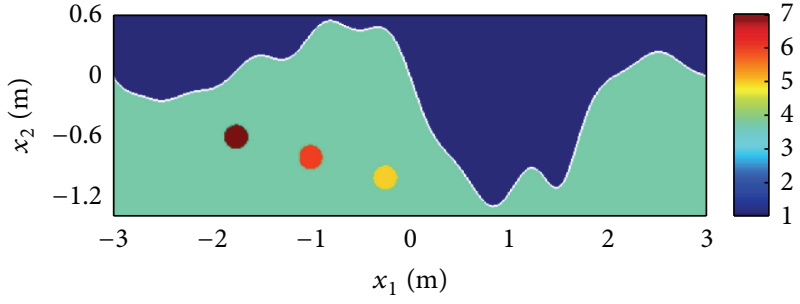

(a)

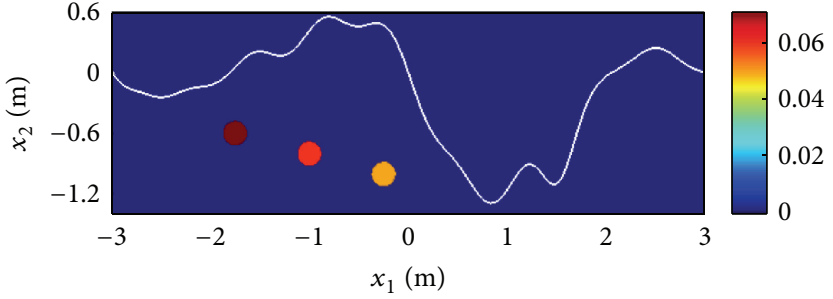

(b)

FiguRE 6: (a) The exact relative permittivity and (b) conductivity profiles of three circular objects and the background having a large roughness.

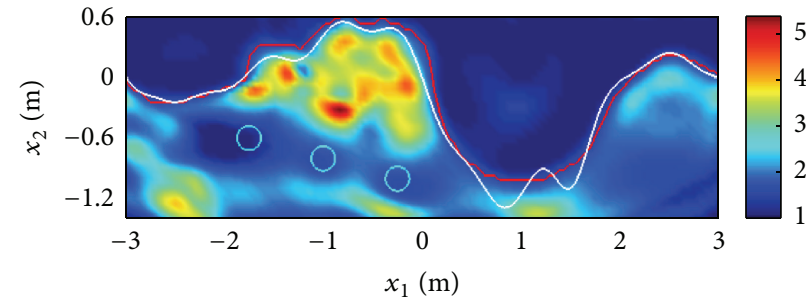

(a)

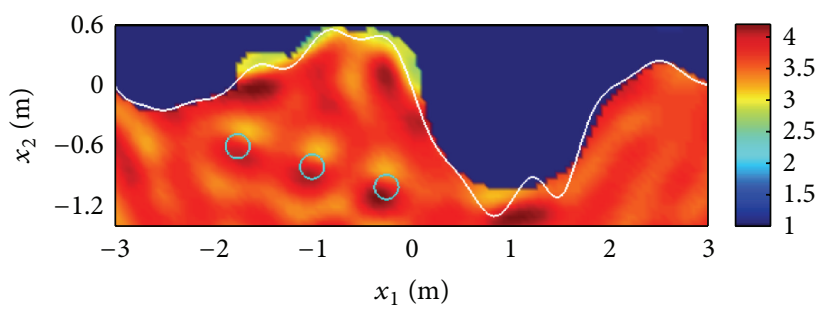

(c)

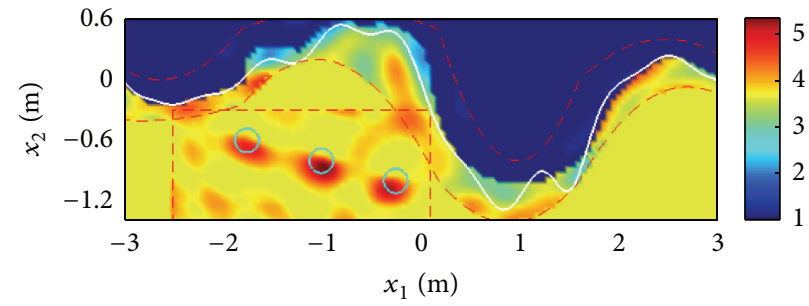

(e)

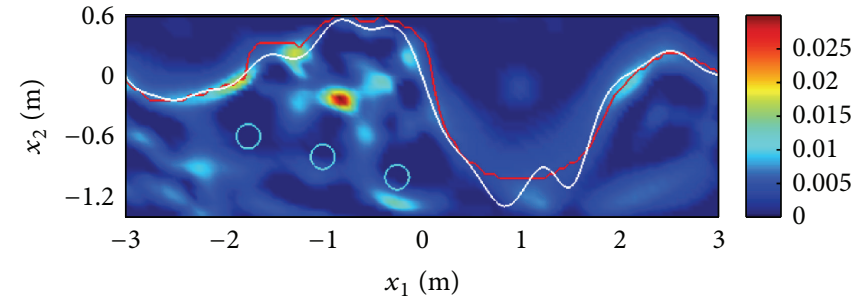

(b)

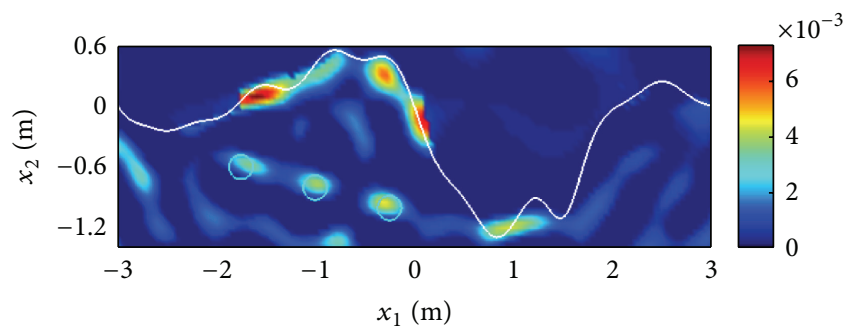

(d)

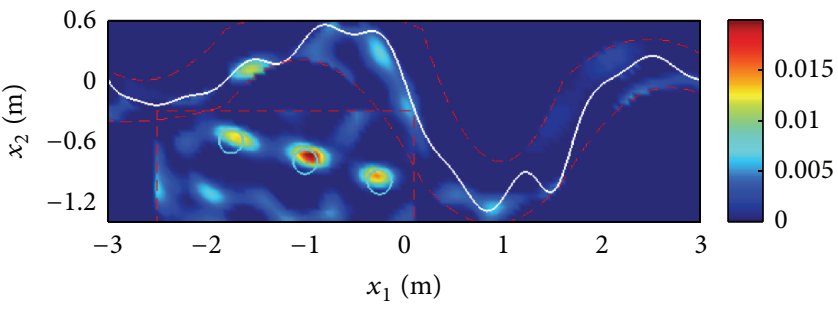

(f)

FIGURE 7: (a) Reconstructed permittivity and (b) conductivity profiles and the determination of the rough surface after the first stage. Final results of permittivity and conductivity profiles ((c)-(d)) for the entire domain and ((e)-(f)) for the narrowed domain.

the rough surface can be approximately determined in the unmodified results of the first stage which are given in Figures $7(\mathrm{a})$ and $7(\mathrm{~b})$. By using the reference model derived from these results, the permittivity and conductivity profiles given in Figures $7(\mathrm{c})$ and $7(\mathrm{~d})$ are reconstructed in the second stage. Although the buried objects are not apparent either in the permittivity or in the conductivity profiles, relatively high values that are concentrated around some specific points are observed on and under the approximate rough surface. Thus, the final inversion is repeated for a narrower domain which is the union of a rectangular region with dimension of $1.1 \times 2.6 \mathrm{~m}(0.55 \lambda \times 1.3 \lambda)$ and a band whose width is averagely about $0.5-0.6 \mathrm{~m}(0.25 \lambda-0.30 \lambda)$. As seen from Figures $7(\mathrm{e})$ and $7(\mathrm{f})$, the quality of the results is significantly improved in this case, and three close objects are distinguished in both the permittivity and conductivity profiles although they are not accurately reconstructed. Note that, in the relative permittivity profile, the estimation errors of the approximate rough surface have been compensated in some degree.

A surface with a relatively high frequency roughness is considered in the third example as seen in Figure 8. Two identical circular objects having radius $0.12 \mathrm{~m}(0.06 \lambda)$ are located in depth of $1 \mathrm{~m}(0.5 \lambda)$ and $0.8 \mathrm{~m}(0.4 \lambda)$. Note that, there are noticeable differences throughout the boundary, 


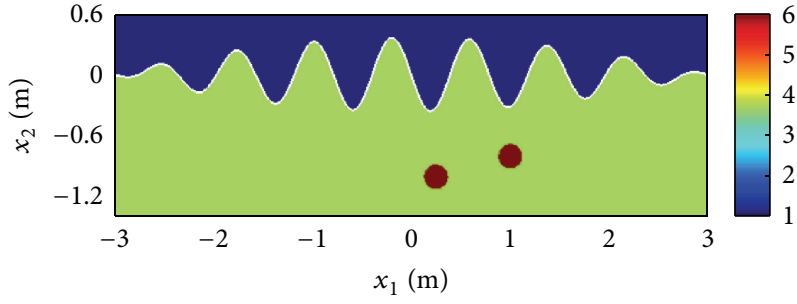

(a)

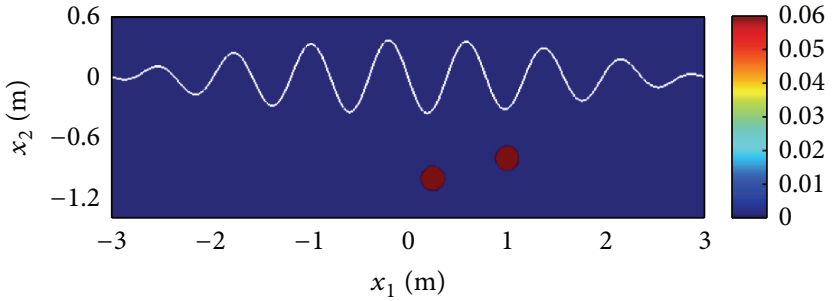

(b)

FIGURE 8: (a) The exact relative permittivity and (b) conductivity profiles of two circular objects and the background having a high frequency roughness.

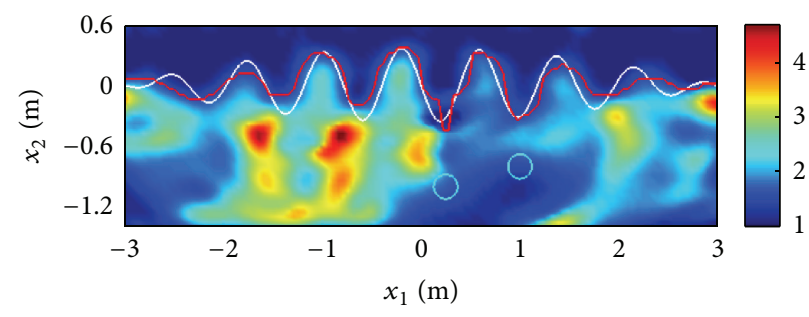

(a)

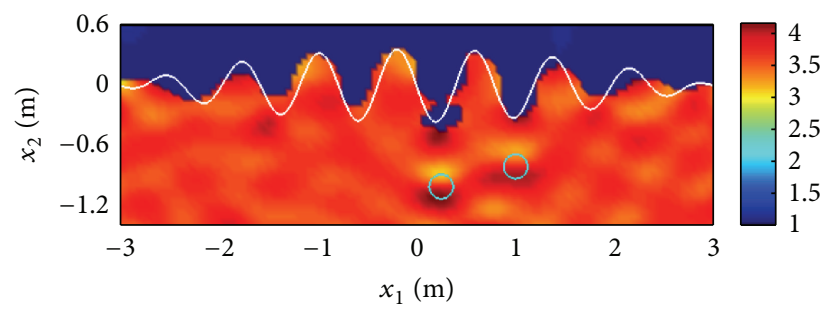

(c)

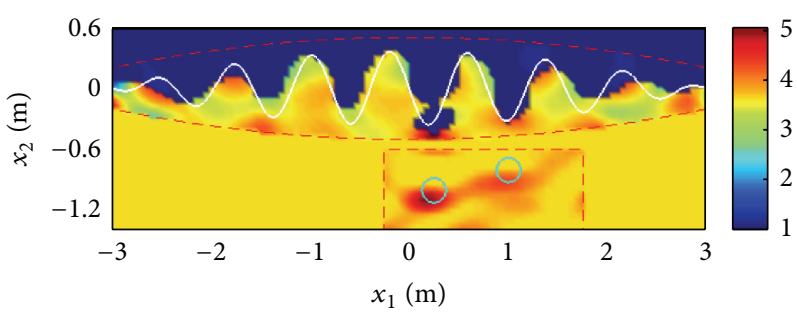

(e)

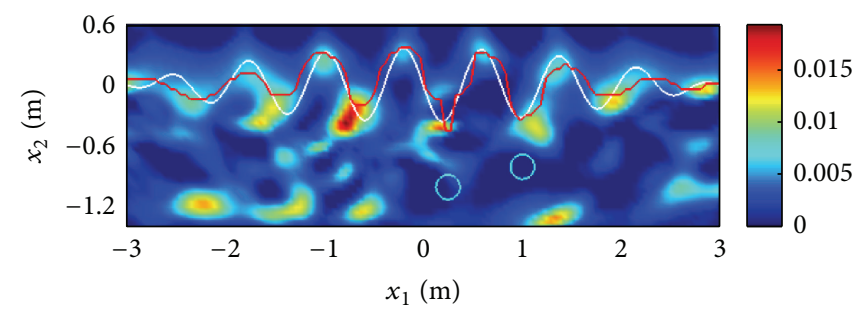

(b)

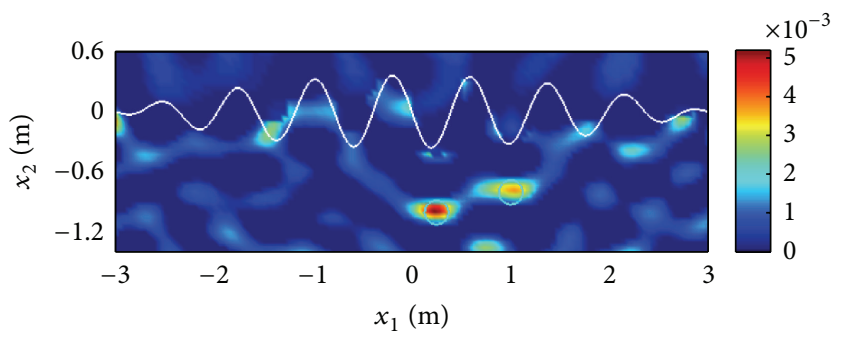

(d)

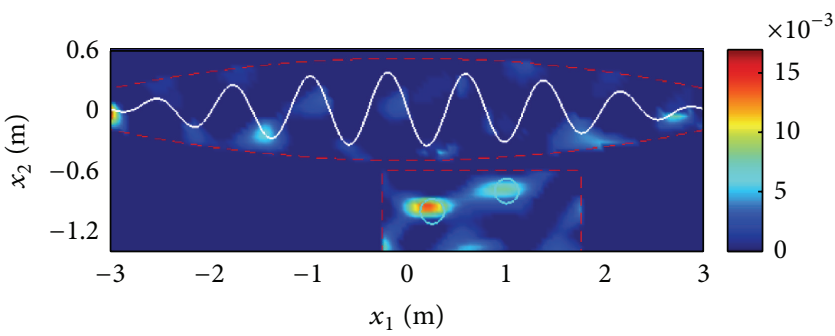

(f)

FIGURE 9: (a) Reconstructed permittivity and (b) conductivity profiles and the determination of the rough surface after the first stage. Final results of permittivity and conductivity profiles ((c)-(d)) for the entire domain and ((e)-(f)) for the narrowed domain.

between the approximate and the actual surfaces given in Figures 9(a) and 9(b). Nevertheless, two objects, whose exact dielectric property values are $\varepsilon_{r, C}=6$ and $\sigma_{C}=0.06 \mathrm{~S} / \mathrm{m}$, can be determined in the results obtained in the entire region. For the narrower domain, although increased dielectric property values are achieved, the visual impact is slightly weakened especially in the conductivity profile. The reason of this weakening is the existence of some surface errors that lead to positive contrasts. When the domain is narrowed, to a region composed of a rectangle with dimension of $0.8 \times 2.0 \mathrm{~m}$
$(0.4 \lambda \times \lambda)$ and a band whose width changes between $0.4 \mathrm{~m}$ $(0.2 \lambda)$ and $1 \mathrm{~m}(0.5 \lambda)$, the reconstructed values of these contrasts are also enhanced. On the other hand, if they are estimated to be slightly below their actual locations, incorrectly high values of dielectric properties on the lower neighborhood of the boundary will be observed.

In the final example, the lower half-space is considered to be composed of a more lossy material that may represent wet soil with constitutive parameters $\varepsilon_{r, 2}=10$ and $\sigma_{2}=10^{-3} \mathrm{~S} / \mathrm{m}$ and a thin bar with dimension of $0.1 \times 1.2 \mathrm{~m}(\lambda / 60 \times \lambda / 5)$ 


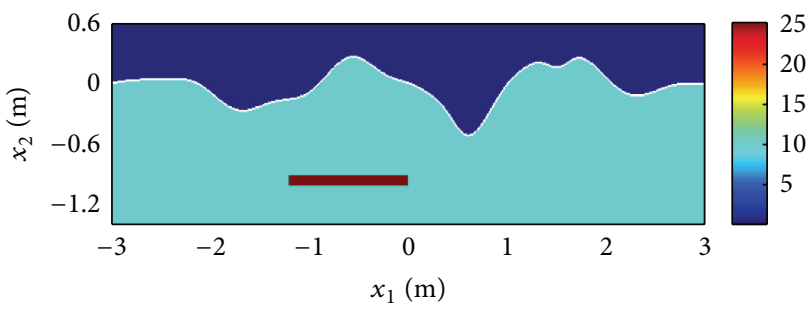

(a)

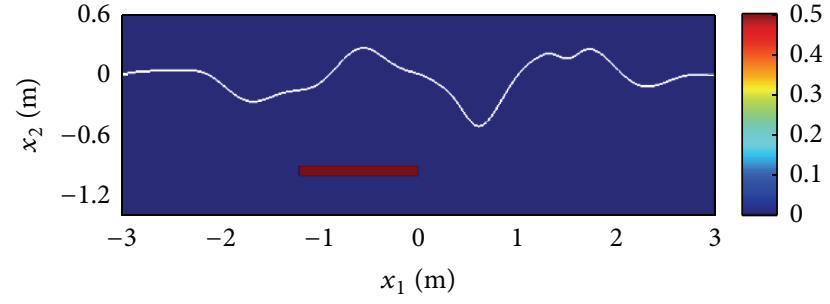

(b)

FIGURE 10: (a) The exact relative permittivity and (b) conductivity profiles of a thin bar and the lossy background.

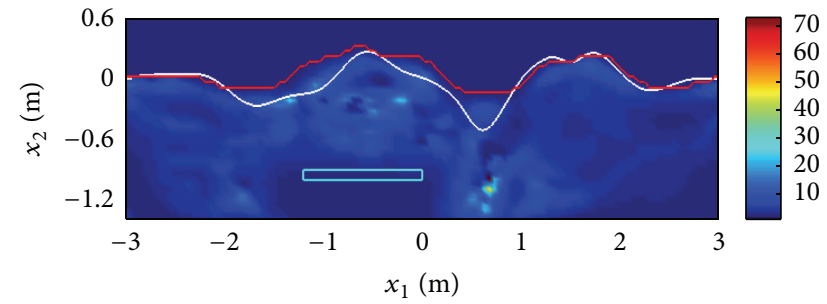

(a)

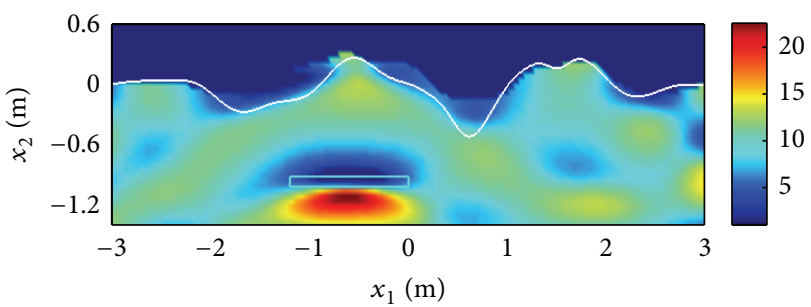

(c)

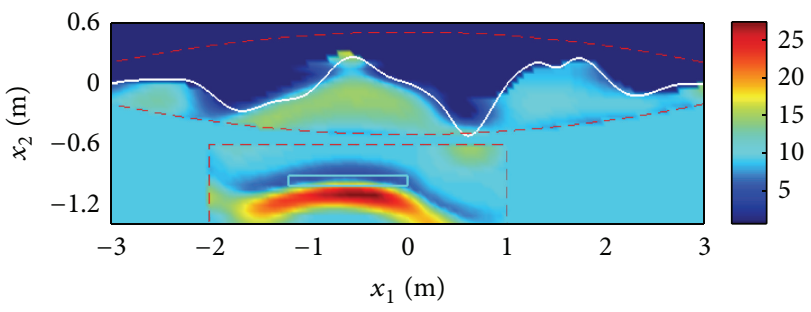

(e)

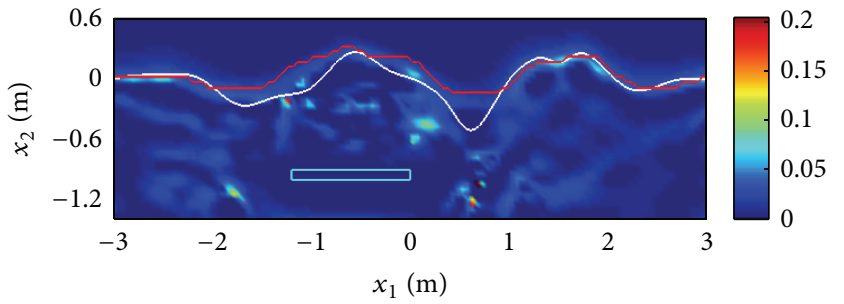

(b)

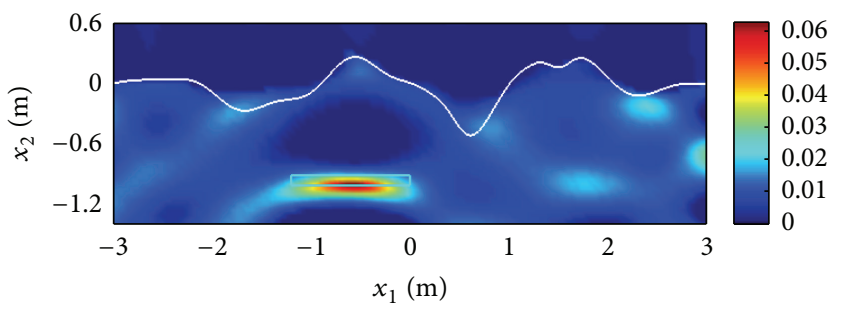

(d)

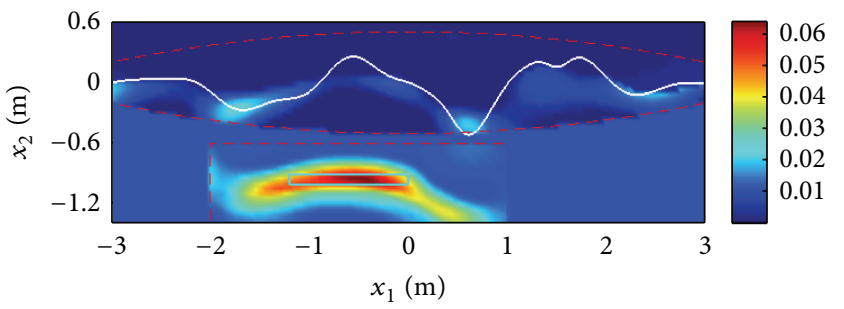

(f)

FIGURE 11: (a) Reconstructed permittivity and (b) conductivity profiles and the determination of the rough surface after the first stage. Final results of permittivity and conductivity profiles ((c)-(d)) for the entire domain and ((e)-(f)) for the narrowed domain.

is buried into it as shown in Figure 10. This bar whose lower edge is in depth of $1 \mathrm{~m}(\lambda / 6)$ has relative permittivity of $\varepsilon_{r, C}=$ 25 and conductivity of $\sigma_{C}=0.5$. The above procedure is repeated but, in the second stage, the frequency is chosen as $50 \mathrm{MHz}$ since penetration of the wave is not sufficient for the detection of the buried object at $150 \mathrm{MHz}$. Thus, $\lambda$ is assumed to be $6 \mathrm{~m}$ for this example. Satisfactory results given in Figure 11 are achieved for both the domain $D$ and the narrower domain consisting of a rectangular region with dimension of $0.8 \times 3.0 \mathrm{~m}(2 \lambda / 15 \times \lambda / 2)$ and a band with changing thickness between $0.4 \mathrm{~m}(\lambda / 15)$ and $1 \mathrm{~m}(\lambda / 6)$. Note that the estimation errors of the rough surface are successfully compensated especially when the reconstruction domain is narrowed.

\section{Conclusions}

We propose a two-stage procedure based on the contrast source inversion (CSI) for the determination of a buried dielectric together with the locally rough surface above it. Despite its extra computational burden, by taking the advantage of nonlinear inversion, the proposed approach yields qualitatively satisfactory results even for multiple objects buried under a surface having a high frequency or large roughness.

Here, we have considered a relatively simple background that consists of two homogeneous half-spaces with known dielectric properties. As a future work, the implementation of the idea of constructing a reference model by preimaging 
the entire region of interest in a frequency-hopping scheme and imposing the a priori known property values to the approximately determined morphology of the background in more challenging problems will be investigated.

\section{Conflict of Interests}

The authors declare that there is no conflict of interests regarding the publication of this paper.

\section{References}

[1] Ö. Özdemir and H. Haddar, "Preprocessing the reciprocity gap sampling method in buried-object imaging experiments," IEEE Geoscience and Remote Sensing Letters, vol. 7, no. 4, pp. 756-760, 2010.

[2] Y. Altuncu, O. Ozdemir, I. Akduman, and A. Yapar, "Imaging of dielectric objects buried under an arbitrary rough surface," in Proceedings of the IEEE International Conference on Geoscience and Remote Sensing Symposium, vol. 6, pp. 2954-2957, IEEE, Denver, Colo, USA, July-August 2006.

[3] Y. Altuncu, F. Akleman, O. Semerci, and C. Ozlem, "Imaging of dielectric objects buried under a rough surface via distorted born iterative method," Journal of Physics: Conference Series, vol. 135, no. 1, Article ID 012006, 2008.

[4] T. U. Gürbuz, B. Aslanyürek, E. P. Karabulut, and I. Akduman, "An efficient nonlinear imaging approach for dielectric objects buried under a rough surface," IEEE Transactions on Geoscience and Remote Sensing, vol. 52, no. 5, pp. 3013-3022, 2014.

[5] O. Cmielewski, T. Hervé, A. Litman, and M. Saillard, "A twostep procedure for characterizing obstacles under a rough surface from bistatic measurements," IEEE Transactions on Geoscience and Remote Sensing, vol. 45, no. 9, pp. 2850-2858, 2007.

[6] R. Firoozabadi, E. L. Miller, C. M. Rappaport, and A. W. Morgenthaler, "Subsurface sensing of buried objects under a randomly rough surface using scattered electromagnetic field data," IEEE Transactions on Geoscience and Remote Sensing, vol. 45, no. 1, pp. 104-117, 2007.

[7] V. Galdi, H. Feng, D. A. Caslañon, W. C. Karl, and L. B. Felsen, "Moderately rough surface underground imaging via short-pulse quasi-ray Gaussian beams," IEEE Transactions on Antennas and Propagation, vol. 51, no. 9, pp. 2304-2318, 2003.

[8] I. Catapano, L. Crocco, and T. Isernia, "A simple twodimensional inversion technique for imaging homogeneous targets in stratified media," Radio Science, vol. 39, no. 1, pp. 148161, 2004

[9] P. M. van den Berg and R. E. Kleinman, "A contrast source inversion method," Inverse Problems, vol. 13, no. 6, pp. 16071620, 1997.

[10] T. J. Cui, W. C. Chew, A. A. Aydiner, and S. Chen, "Inverse scattering of two-dimensional dielectric objects buried in a lossy earth using the distorted born iterative method," IEEE Transactions on Geoscience and Remote Sensing, vol. 39, no. 2, pp. 339-346, 2001.

[11] W. C. Chew and J. H. Lin, "A Frequency-hopping approach for microwave imaging of large inhomogeneous bodies," IEEE Microwave and Guided Wave Letters, vol. 5, no. 12, pp. 439-441, 1995.

[12] R. F. Bloemenkamp, A. Abubakar, and P. M. van den Berg, "Inversion of experimental multi-frequency data using the contrast source inversion method," Inverse Problems, vol. 17, no. 6, pp. 1611-1622, 2001.

[13] M. I. Aksun, "A robust approach for the derivation of closedform Green's functions," IEEE Transactions on Microwave Theory and Techniques, vol. 44, no. 5, pp. 651-658, 1996.

[14] J. H. Richmond, "Scattering by a dielectric cylinder of arbitrary cross section shape," IEEE Transactions on Antennas and Propagation, vol. 13, no. 3, pp. 334-341, 1965. 

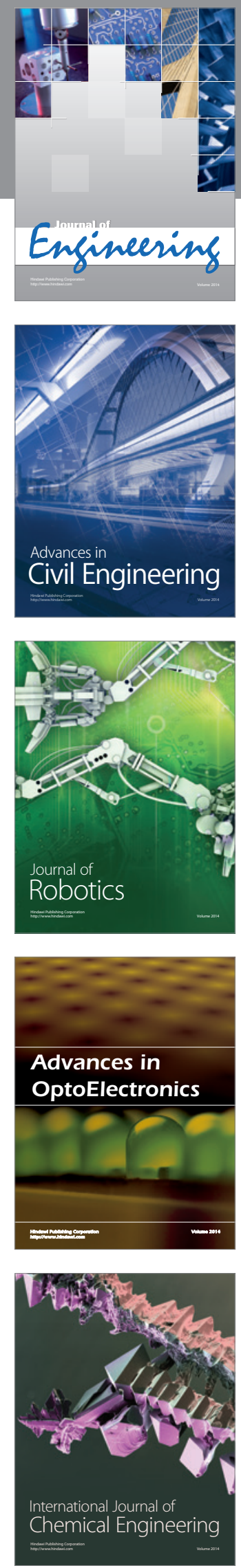

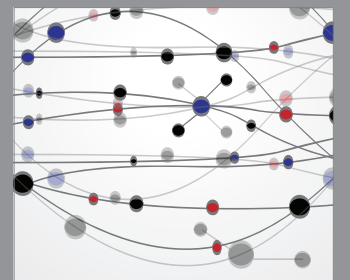

The Scientific World Journal
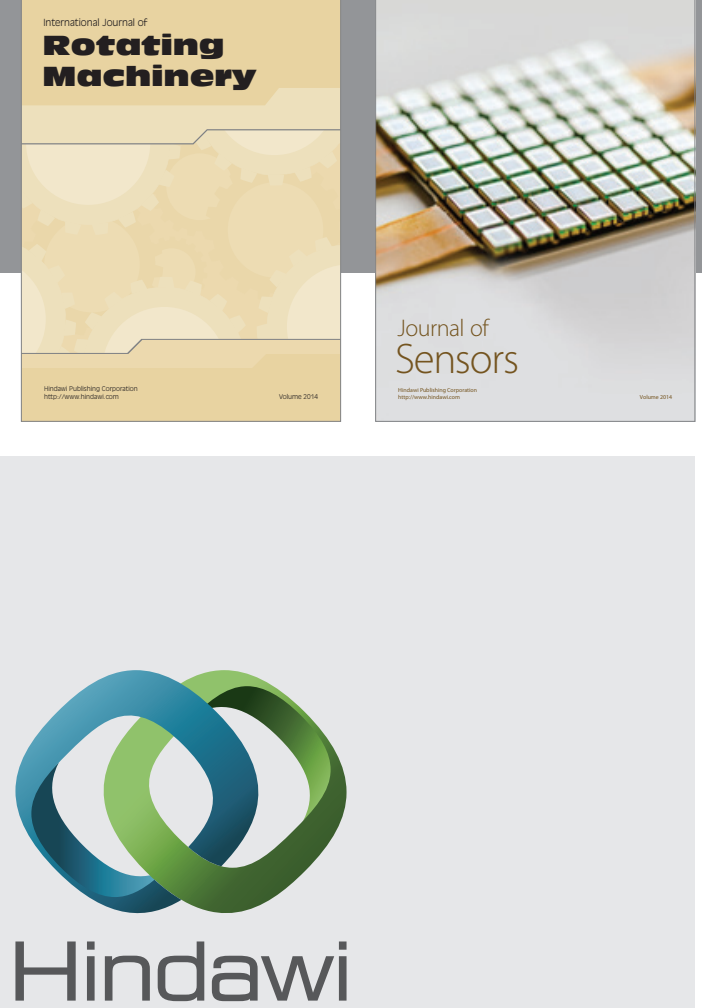

Submit your manuscripts at http://www.hindawi.com
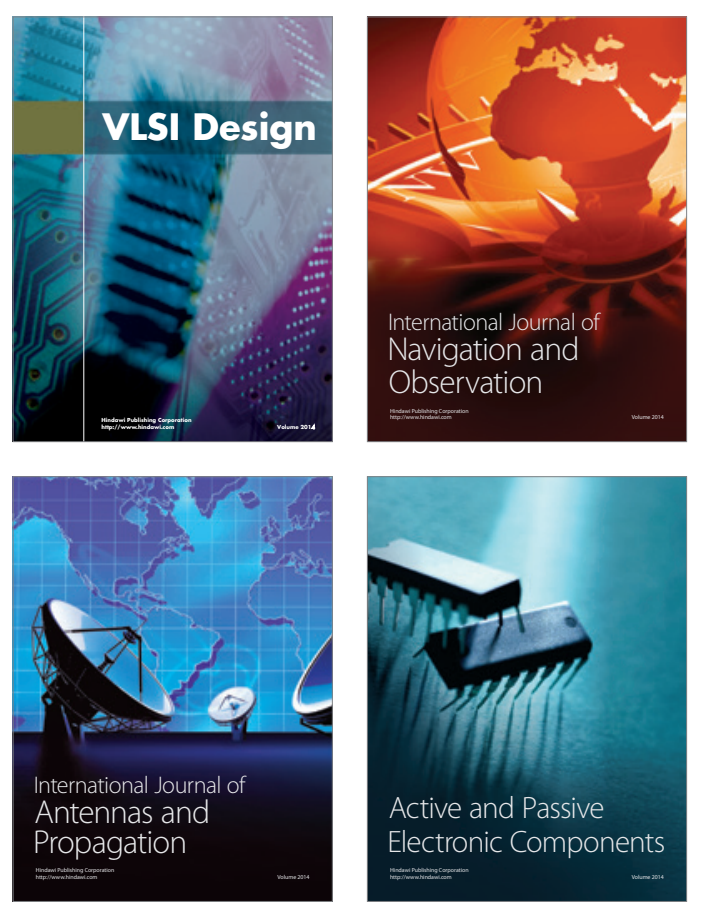
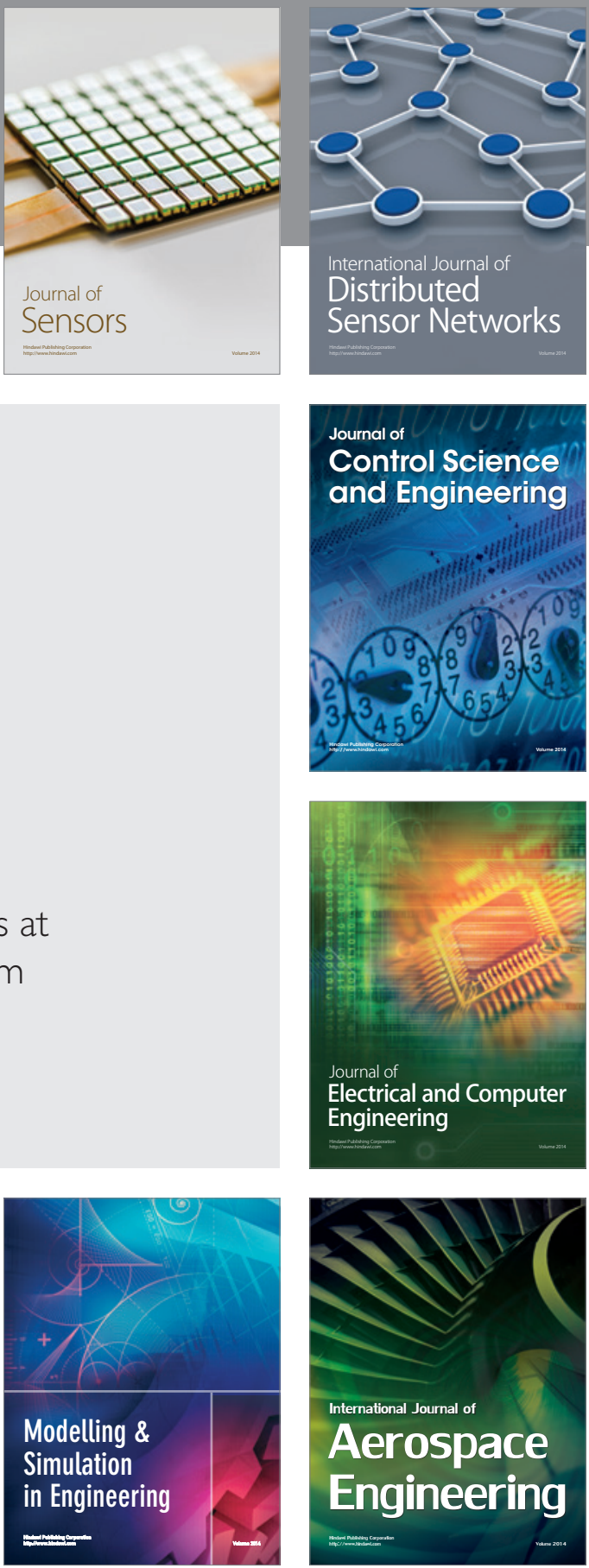

Journal of

Control Science

and Engineering
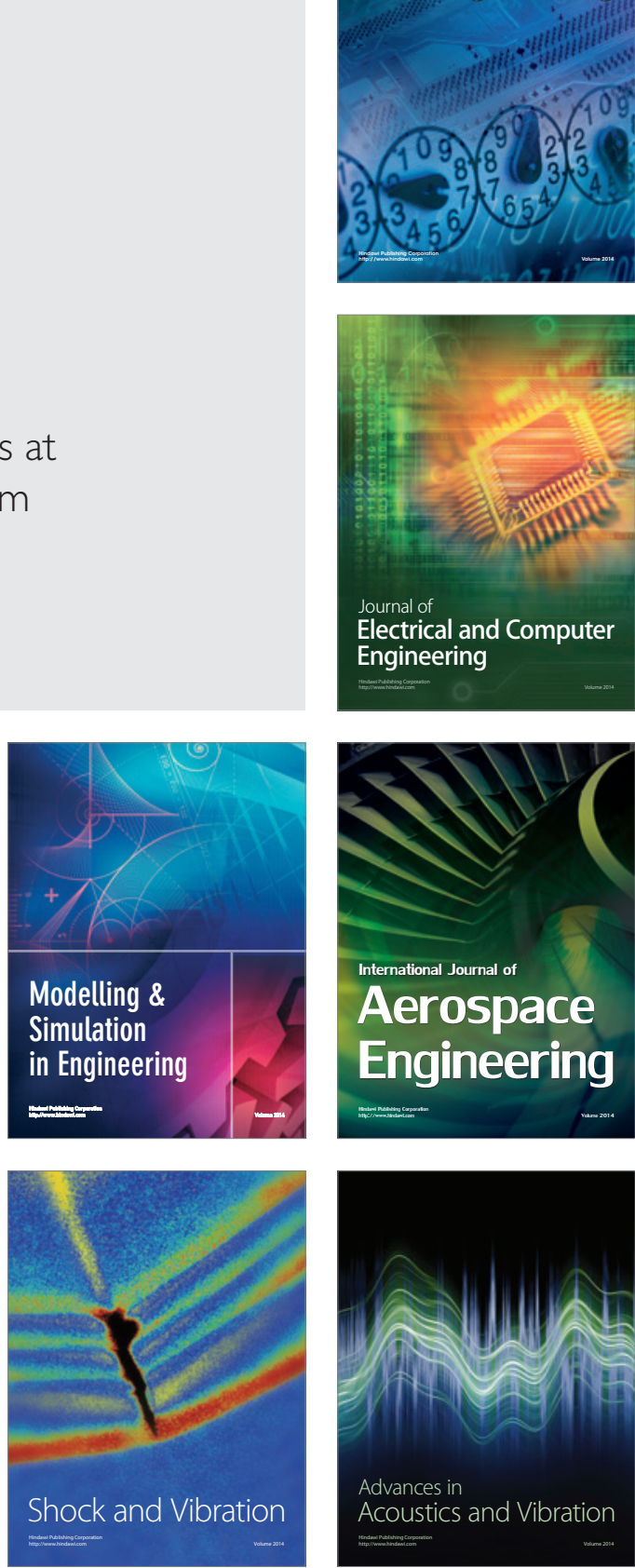